\title{
A remark on arithmetic equivalence and the normset
}

\author{
by \\ Jim Coykendall (Fargo, ND)
}

1. Introduction. Number fields with the same zeta function are said to be arithmetically equivalent. Arithmetically equivalent fields share much of the same properties; for example, they have the same degrees, discriminants, number of both real and complex valuations, and prime decomposition laws (over $\mathbb{Q}$ ). They also have isomorphic unit groups and determine the same normal closure over $\mathbb{Q}[6]$. Strangely enough, it has been shown (for example [4], or more recently [6] and [7]) that this does not imply that arithmetically equivalent fields are isomorphic. Just recently, B. De Smit and R. Perlis [3] showed that arithmetically equivalent fields do not even necessarily have the same class number.

In this short note we take this recent result of De Smit and Perlis, and a well-known fact from algebraic number theory and use them to show that the integral normset (that is, the set of integral norms from a ring of algebraic integers to $\mathbb{Z}$ ) uniquely determines a larger class of extensions of $\mathbb{Q}$ than the splitting set does. (In this paper, we use the standard convention that "equality of splitting sets" means that the symmetric difference is finite. That is to say, if $X$ and $Y$ are sets of prime ideals, then we say that they are equal if their symmetric difference is finite.)

Good background information on the splitting set can be obtained in [1] or [5], and information on the normset can be found in [2].

2. The splitting set and the normset. In this paper, $K$ and $L$ will denote arithmetically equivalent field extensions of $\mathbb{Q}$. We will denote the rings of integers as $S$ and $T$ respectively, and their normsets (with respect to $\mathbb{Z}$ ) will be denoted by $N_{S}$ and $N_{T}$. We recall that the splitting set of an extension (or its corresponding ring of integers) is the set of primes in the base field that have a degree-one factor in the extension. It is well known that a normal extension of a number field $F$ is determined uniquely among

2000 Mathematics Subject Classification: Primary 11R04, 11R42; Secondary 11M06. 
all finite extensions of $F$ by its splitting set (see the exercise in Cassels and Frohlich [1]). An easy corollary to this fact is that the normset also determines the field extension in this case. To see this merely note that the splitting set is the set of primes in the base field that occur to the first power in the normset.

So in the case of Galois extensions, we see that much information is encoded in both the splitting set and the normset. Intuitively it would seem that in general, more information would be contained in the normset, and our main result will show that this is true. In fact, the examples given show that arithmetic equivalence does not imply (integral) normset equivalence, and integral normset equivalence does not imply that the fields in question are isomorphic. A consequence of this is that there are pairs of fields that can be distinguished by integral normset equivalence although they cannot be distinguished by the way that prime numbers split in the fields.

Theorem 2.1. Let $K=\mathbb{Q}(\sqrt[8]{-15}), L=\mathbb{Q}(\sqrt[8]{-240})$. $K$ and $L$ are arithmetically equivalent fields with rings of integers $S$ and $T$ possessing different normsets.

Remark 2.2. Perlis and De Smit showed in [3] that the respective rings of integers $S$ and $T$ have different class numbers.

The strategy of this proof will be to show that there is no element of $T$ that has norm 15 (of course the element $\sqrt[8]{-15}$ of $S$ has norm 15). To establish the nonexistence of an element of $T$ of norm 15 , we first need a couple of lemmas.

Lemma 2.3. In both $K$ and $F$, the rational primes 3 and 5 are totally ramified.

Proof. It is enough to notice that the polynomials $x^{8}+15$ and $x^{8}+240$ are both 3 and 5 Eisensteinian. The result then follows from Theorem 4.12 of [5].

Lemma 2.4. The unit group of $T$ is generated by

$$
\begin{aligned}
\left\{-1, \frac{r^{6}+2 r^{4}-4 r^{2}-56}{16}, \frac{r^{7}-2 r^{6}+2 r^{5}-4 r^{3}+8 r^{2}-8 r+64}{64},\right. & \left.\frac{r^{7}-2 r^{5}+4 r^{4}-4 r^{3}-32 r^{2}+8 r-16}{64}\right\}
\end{aligned}
$$

with $r$ a root of $x^{8}+240$.

Proof. These units were found in [3] using the computer system PARI, which lists them as fundamental units.

Proof of Theorem 2.1. Lemma 2.3 implies that $\alpha=\sqrt[4]{-15}$ is the unique (up to a unit factor) element of $T$ of norm 225. If there is an element $z \in T$ 
such that $N(z)=15$ then $z^{2}=u \alpha$, with $u$ denoting a unit of $T$. Therefore we have the equations

$$
z=\sqrt{u \alpha}=\sqrt{u} \sqrt[8]{-15}=\sqrt{u / 2} \sqrt[8]{-15} \sqrt{2}=\sqrt{u / 2} \sqrt[8]{-240}
$$

Hence, we conclude that $u / 2$ is a square in $L$ (and of course $2^{2 n-1} u$ is also a square and is in $T$ for $n \geq 1$ ). Let $\mathcal{P}$ be the (unique) prime lying over the rational prime 5 in $T$. By Lemma $2.3, T / \mathcal{P}$ is isomorphic to $\mathbb{Z} / 5 \mathbb{Z}$. So, reducing the units from Lemma 2.4 modulo 5 (and letting $r$ be equivalent to 0 ), we see that all of the fundamental units are equivalent to $\pm 1 \bmod 5$. Therefore any unit of $T$ times an odd power of 2 is equivalent to 2 or 3 mod 5 and is therefore a nonsquare mod 5 . Hence $u / 2$ cannot be a square in $L$, and we have a contradiction.

EXAMPLE 2.5. The natural question that one may ask is: "Do all nonisomorphic fields have different normsets?" The answer, unfortunately, is no. Consider the nonisomorphic but arithmetically equivalent fields $\mathbb{Q}(\sqrt[8]{-3})$ and $\mathbb{Q}(\sqrt[8]{-48})$. It is shown in $[8]$ that both have class number 1 . Hence the normsets of both fields are generated by the norms of all the primes, and since the prime decomposition laws are the same in arithmetically equivalent fields, these two fields must have identical normsets.

REMARK 2.6. The results of this paper show that, in general, the normset is better at distinguishing nonisomorphic fields than the splitting set is. Example 2.5, however, dramatically points out the limitations of this approach. One would like to find "the best" large class of fields that are determined uniquely by their normsets. Because of the somewhat limited knowledge on the distribution of primes in the non-Galois case, a satisfactory answer to this question has still not been realized, even in the isomorphic class group case.

Acknowledgements. The author would like to extend his gratitude to Dr. Robert Perlis for helpful conversations and inspirations.

\section{References}

[1] J. W. S. Cassels and A. Frohlich, Algebraic Number Theory, Academic Press, London, 1967.

[2] J. Coykendall, Normsets and determination of unique factorization in rings of algebraic integers, Proc. Amer. Math. Soc. 124 (1996), 1727-1732.

[3] B. De Smit and R. Perlis, Zeta functions do not determine class numbers, Bull. Amer. Math. Soc. 31 (1994), 213-215.

[4] F. Gassmann, Bemerkungen zu der vorstehenden Arbeit von Hurwitz, Math. Z. 25 (1926), 124-143.

[5] W. Narkiewicz, Elementary and Analytic Theory of Algebraic Numbers, Springer and Polish Sci. Publ., Warszawa, 1990. 
[6] R. Perlis, On the equation $\zeta_{K}(s)=\zeta_{K^{\prime}}(s)$, J. Number Theory 9 (1977), 342-360.

[7] -, On the class numbers of arithmetically equivalent fields, ibid. 10 (1978), 489-509.

[8] R. Perlis and A. Schinzel, Zeta functions and the equivalence of integral forms, J. Reine Angew. Math. 309 (1979), 176-182.

Department of Mathematics

North Dakota State University

Fargo, ND 58105-5075, U.S.A.

E-mail: coykenda@plains.nodak.edu 